\title{
Pseudotyped Lentiviral Vectors: One Vector, Many Guises
}

\author{
Alok V. Joglekar ${ }^{1, \dagger}$ and Salemiz Sandoval ${ }^{1, \dagger}$ \\ ${ }^{1}$ Department of Biology and Biological Engineering, California Institute of Technology, Pasadena, California. \\ †These authors contributed equally to this work.
}

\begin{abstract}
Viruses have evolved specialized molecular mechanisms to transfer their genome efficiently into host cells. Viruses can be repurposed into viral vectors to achieve controlled gene transfer to desired cells. One of the most popular classes of vectors, lentiviral vectors $(\mathrm{LVs})$, transduce mammalian cells efficiently. LVs are pseudotyped with various heterologous viral envelopes to alter their tropism. While the most common example is the envelope glycoprotein from vesicular stomatitis virus (VSVG), many other viral proteins have also been used. Pseudotyping LVs with a diverse set of naturally occurring or engineered viral envelopes has allowed targeted transduction of specific cell types. Many exciting studies are further uncovering new specificities and shortcomings of pseudotyped LVs. These studies will expand the toolbox to make LVs that cater to the specific requirements of transduction. This review provides a comprehensive overview of various viral envelope pseudotypes used with LVs, their specificities, advantages, and drawbacks.
\end{abstract}

Keywords: lentiviral vector envelopes, pseudotypes, pseudotyped lentiviral vect

\section{LENTIVIRAL VECTORS}

VIRAL VECTORS HAVE LONG been the tool of choice for transferring genetic material to cells because they have evolved specialized molecular mechanisms of gene transduction. One of the most prominent types of vectors, Lentiviral Vectors (LVs), are often the preferred viral delivery systems for various purposes. LVs have several advantages over other gene transfer vectors: (1) they integrate irreversibly into the host genome, therefore providing sustained transgene expression that heritable upon cell division; (2) they are engineered to package relatively large payloads; (3) they show low immunogenicity due to absence of all viral coding genes; (4) they are extensively engineered to increase biosafety; and (5) they can be pseudotyped with numerous heterologous envelope glycoproteins. LV design evolved conceptually from oncoretroviral vectors, which were first used to introduce genetic material into cells. Originally, these vectors were derived from the Moloney Murine Leukemia Virus (MoMLV). They were replication competent viruses that could not infect nondividing cells, and were susceptible to silencing and insertional oncogenesis. A solution to transduce nondividing cells efficiently was presented when LVs were first developed in the 1990 s. $^{1-3}$ Lentiviruses are a family of retroviruses that are capable of infecting and integrating into nondividing cells. LVs were first constructed by "gutting" the Human Immunodeficiency Virus (HIV) genome and supplementing it with HIV polyproteins in trans. ${ }^{3}$ Since then, a series of modifications to the vector genome have helped optimize vector design to transduce nondividing cells while improving the production and safety of the vectors.

\section{FROM CD4 TROPISM OF HIV TO BROAD TROPISM OF VESICULAR STOMATITIS VIRUS}

The HIV envelope glycoprotein, a class I fusion protein, is generated by cleavage of the precursor gp160 in two subunits: outer membrane protein gp120, and inner membrane protein gp41. The HIV envelope consists of three gp120/gp41 heterodimers. HIV binds to the surface of cells by binding to its primary cellular surface receptor: CD4 and co-receptors CXCR4 or CCR5. ${ }^{4-6}$ Binding of gp120 to CD4 and CXCR4/CCR5 leads to a change

${ }^{*}$ Correspondence: Dr. Alok Vishnu Joglekar, Department of Biology and Biological Engineering, California Institute of Technology, Pasadena, CA 91125. E-mail: alokvj@caltech.edu 
in conformation of gp41, facilitating target cell membrane fusion and viral entry. Because the HIV envelope preferentially recognizes the human CD4 receptor, early infections necessitated the expression of CD4 on the surface of target cells, limiting the tropism to CD4+ T cells and monocytes.

To circumvent this problem, Burns et al. pseudotyped the early onco-viral vectors based on $\mathrm{Mu}$ rine Leukemia Virus (MLV) with the glycoprotein of the Vesicular Stomatitis Virus (VSV), VSVG. ${ }^{7}$ VSV is a member of the Rhabdoviridae family, which consists of single-stranded, negative-sense RNA viruses. VSVG is a trimeric protein that binds to its cellular receptor and facilitates endocytosis of the virus. Acidification (decrease in $\mathrm{pH}$ ) of the endosomes triggers conformational changes in VSVG that allow fusion with the cell membrane. Phosphatidylserine and low-density lipoprotein receptor (LDLR) have been considered as the viral receptor for VSVG. ${ }^{8}$ VSVG viral pseudotyping has now become the mainstay in lentiviral production. ${ }^{7}$ VSVG-LVs have extremely broad tropism and can transduce many cell types. VSVG pseudotyped LVs are also stable and can withstand ultracentrifugation, allowing generation of high-titer LV preparations. These two properties of the VSVG envelope have facilitated transduction of very difficult to transduce cells such as stem cells and neurons. ${ }^{9,10}$ However, there are a few shortcomings to using the VSVG envelope. VSVG-LVs transduce resting lymphocytes inefficiently, primarily because of low LDLR expression levels. ${ }^{11}$ At high concentrations, VSVG can be highly toxic to both producer cells and the transduced cells. Therefore, generation of packaging cell lines has been difficult, as sustained VSVG expression is not viable. Nevertheless, stable viral packaging cell lines that express VSVG from inducible promoters have been used successfully to package vectors. ${ }^{12-14}$ Another limitation is that VSVG-LVs are inactivated by serum, making their in vivo administration ineffective. ${ }^{15}$ Additionally, because of their broad tropism, VSVG-LVs transduce unwanted cells after systemic in vivo delivery. ${ }^{16}$ Due to these reasons, there has been a significant body of research exploring alternative envelopes for pseudotyping LVs to achieve: (1) targeted cell tropism, including hard-to-transduce cells, (2) low cytotoxicity during transduction and vector packaging, and (3) resistance to serum inactivation and ultracentrifugation. The subsequent sections provide an overview of various strategies used to overcome the limitations of VSVG. These results are summarized in Table 1.

Some of the research in pseudotyping LVs has examined closely related vesiculoviruses, such as
Chandipura (CNVG) and Piry (PRVG) strains of VSV. These viruses, while related to VSV, have different cellular tropisms. Like VSVG, these envelopes are able to withstand high-speed centrifugation and are more resistant to serum inactivation. ${ }^{17}$ However, compared to VSVG-LVs, they do not transduce blood cells as efficiently. ${ }^{17}$ CNVG-LVs, for example, are more neurotropic, showing higher transduction into neuroblastoma cells compared to VSVG. VSVG-LVs outperform CNVG-LVs and PRVG-LVs in transduction of CD4+ and CD8+ T cells, NK cells, and CD34+ hematopoietic stem cells (HSCs). ${ }^{18,19}$ Envelope from another closely related virus, Cocal virus, exhibits broad tropism similar to VSVG. Cocal-LVs show higher transduction efficiencies in human and nonhuman primate CD34+ HSCs and CD4+ T cells, and higher resistance to serum inactivation compared to VSVG-LVs. ${ }^{20}$ Additionally, several studies have attempted to engineer VSVG to increase its serum stability. VSVG variants generated by mutagenesis libraries that are resistant to a panel of human and animal sera and that are thermostable were identified by Schafer et al. ${ }^{21}$

\section{RETROVIRAL ENVELOPES}

Perhaps the most "obvious" extension of to pseudotyping LVs is to use envelopes from their close relatives in the Retroviridae family. Indeed, numerous studies have demonstrated pseudotyping of LVs with envelopes from other retroviruses such as MLV, ${ }^{22-24}$ Gibbon Ape Leukemia Virus (GALV), ${ }^{25,26}$ Feline Leukemia Virus (RD114), ${ }^{26-29}$ Amphotropic Retrovirus (Ampho), ${ }^{30-32}$ 10A1 MLV (10A1), ${ }^{33,34}$ and Ecotropic retrovirus (Eco). ${ }^{31,34,35}$ Retroviruses are generally conserved in their genome and virion structure. Retroviral envelope glycoproteins attach to their receptors and fuse directly to the host-cell membrane. Retroviral envelope glycoproteins are synthesized as a single polypeptide, which is cleaved by the viral protease into two subunits: surface (SU) and transmembrane (TM). Fully mature envelopes are made up of heterotrimers of SU and TM. SU subunits are heavily glycosylated, which facilitates binding to their receptors. TM proteins consist of three domains: an N-terminal ectodomain, a membrane-spanning domain, and a cytoplasmic tail. SU-receptor binding triggers a conformational change in the TM protein, allowing insertion of the ectodomain into the host-cell membrane, leading to fusion. ${ }^{36}$ The membranespanning domain anchors the viral and cellular membranes, whereas cytoplasmic tails interact with the matrix (MA) protein. Despite considerable sequence variability, the organizational structure 
Table 1. Summary of viral envelopes described in this study and their tropisms

\begin{tabular}{|c|c|c|c|c|}
\hline Virus & Envelope & Receptor & Cell type specificity & Reference \\
\hline Human immunodeficiency virus (HIV) & gp160 (gp41 and gp120) & CD4, CCR5/CXCR4 & T cells, monocytes & $4-6$ \\
\hline \multicolumn{5}{|l|}{ Retroviruses } \\
\hline Murine leukemia virus (MLV) & gp70 (SU and TM) & mCAT-1 & Hematopoietic cells, fibroblasts & $22-24$ \\
\hline Gibbon ape leukemia virus (GALV) & gp70 (SU and TM) & Pit1 & Hematopoietic cells & 25,26 \\
\hline Feline leukemia virus (RD114) & gp70 (SU and TM) & SLC1A5, hASCT2 & Hematopoietic cells & $26-29$ \\
\hline Amphotropic retrovirus (Ampho) & gp70 (SU and TM) & RAM1 & Hematopoietic cells & 33,34 \\
\hline 10A1 MLV (10A1) & gp70 (SU and TM) & Pit1, Pit 2 & Hematopoietic cells & 33,34 \\
\hline Ecotropic retrovirus (Eco) & gp70 (SU and TM) & Rec1 & Hematopoietic cells & $31-35$ \\
\hline Baboon ape leukemia virus (BaEV) & gp70 (SU and TM) & hASCT2 & Hematopoietic cells & 38,39 \\
\hline \multicolumn{5}{|l|}{ Paramyxoviruses } \\
\hline Measles virus (MV) & $\mathrm{H}$ and $\mathrm{F}$ & CD46, SLAM & Lymphocytes, dendritic cells & $41-44$ \\
\hline Nipah virus (NiV) & $\mathrm{H}$ and $\mathrm{F}$ & EphrinB2 & $\begin{array}{l}\text { Embryonic stem cells, hematopoietic } \\
\text { stem cells }\end{array}$ & 49,50 \\
\hline \multicolumn{5}{|l|}{ Rhabdoviruses } \\
\hline Rabies virus (RabV) & G & P75NTR, NCAM, nAchR & Neural cells & $51,57-60$ \\
\hline Mokola virus (MOKV) & G & Not known & Neural cells & 61,62 \\
\hline Ebola Zaire virus (EboZ) & G & NPC1, TIM1 & hematopoietic cells, lung epithelial cells & 62,63 \\
\hline $\begin{array}{l}\text { Lymphocytic choriomeningitis } \\
\text { virus (LCMV) }\end{array}$ & GP1 and GP2 & $\alpha$-dystroglycan & Dendritic cells, neural cells, hepatocytes & $64-67$ \\
\hline Baculovirus & GP64 & Heparan sulfate, phospholipids & Fibroblasts, hepatocytes, epithelial cells & $68-70$ \\
\hline \multicolumn{5}{|l|}{ Alphaviruses } \\
\hline Chikungunya virus (CHIKV) & $\mathrm{E} 1$ and $\mathrm{E} 2$ & $\begin{array}{l}\text { Potential involvement of heparin } \\
\text { sulfate and integrins }\end{array}$ & $\begin{array}{l}\text { Hematopoietic cells, fibroblasts, } \\
\text { hepatocytes }\end{array}$ & $72,73,78,79$ \\
\hline Ross River virus (RRV) & $\mathrm{E} 1$ and E2 & $\begin{array}{l}\text { Potential involvement of heparin } \\
\text { sulfate and integrins }\end{array}$ & $\begin{array}{l}\text { Fibroblasts, neuroglial cells, hepatocytes, } \\
\text { Kupffer cells }\end{array}$ & 72 \\
\hline Semliki Forest virus (SFV) & $\mathrm{E} 1$ and E2 & $\begin{array}{l}\text { Potential involvement of heparin } \\
\text { sulfate and integrins }\end{array}$ & $\begin{array}{l}\text { Fibroblasts, neuroglial cells, hepatocytes, } \\
\text { Kupffer cells }\end{array}$ & 72 \\
\hline Sindbis virus (SV) & E1 and E2 & $\begin{array}{l}\text { Potential involvement of heparin } \\
\text { sulfate and integrins }\end{array}$ & Hematopoietic cells, fibroblasts, hepatocytes & 77 \\
\hline $\begin{array}{l}\text { Venezualan equine encephalitis } \\
\text { virus (VEEV) }\end{array}$ & E1 and E2 & $\begin{array}{l}\text { Potential involvement of heparin } \\
\text { sulfate and integrins }\end{array}$ & Fibroblasts, hepatocytes & 77 \\
\hline $\begin{array}{l}\text { Western equine encephalitis } \\
\text { virus (WEEV) }\end{array}$ & E1 and E2 & $\begin{array}{l}\text { Potential involvement of heparin } \\
\text { sulfate and integrins }\end{array}$ & & 77 \\
\hline \multicolumn{5}{|l|}{ Orthomyxoviruses } \\
\hline Influenza A-D & HA & Sialic acid & Airway epithelia & 80 \\
\hline Fowl Plague Virus (FPV) & $\mathrm{HA}$ & Sialic acid & Airway epithelia & 81 \\
\hline \multicolumn{5}{|l|}{ Vesiculoviruses } \\
\hline Vesicular stomatitis virus & VSV-G & LDLR & Broad tropism & 7,8 \\
\hline Chandipura virus and Piry virus & CNV-G and PRV-G & not known & Neural cells & $17-19$ \\
\hline
\end{tabular}

of retroviral envelopes is conserved. While theoretically modular, combinations of vectors with heterologous envelope glycoproteins are not always compatible. For instance, Ampho or 10A1 pseudotype HIV-1-based LVs efficiently, whereas wild-type GALV does not. In contrast, all three envelops, Ampho, 10A1, and GALV, can pseudotype MLV-based retroviral vectors efficiently. ${ }^{31}$ Lack of compatibility with a heterologous vector is dependent upon the cytoplasmic tails of the envelopes. In some cases, co-expression of heterologous gag proteins hinders the processing of envelope or its colocalization with the capsid. ${ }^{36}$ These deficiencies can often be rescued by partially replacing the cytoplasmic tail of the envelope with that from another virus. For instance, GALV envelope with either truncated or chimeric C-terminal show $10^{4}$ fold enhancement in lentiviral titers due to effi- cient processing. Simian immunodeficiency virusbased LVs pseudotyped with RD114 envelop are not packaged efficiently. Partial replacement of the TM region with that from MLV (RD114-TR) results in efficient co-localization of envelope and capsid, leading to faster maturation of viral particles and efficient virion packaging. ${ }^{36}$

The diverse tropisms offered by various retroviral envelopes combined with the ability of lentiviruses to transduce nondividing cells and lower risk of insertional mutagenesis makes a compelling argument for RV-pseudotyped LVs. Indeed, LVs pseudotyped with heterologous retroviral envelopes have been used extensively. Notably, LVs pseudotyped with MLV and Eco envelope transduce murine cells efficiently. Of particular importance to gene therapy are LVs pseudotyped with GALV or RD114-TR envelopes. ${ }^{27,29}$ Several studies 
have shown that GALV/RD114-TR pseudotyped vectors can transduce human CD34+ hematopoietic stem/progenitor cells at high levels and with minimal cytotoxicity. ${ }^{25,37}$ Moreover, these envelope proteins are sufficiently stable to allow vector concentration by ultracentrifugation. Another envelope of interest is the Baboon Endogenous Retrovirus Envelope (BaEV), which has been shown to pseudotype LVs effectively for gene transfer into human HSCs. The BaEV envelope uses two surface receptors, the neutral amino acid transporters ASCT-1 and ASCT-2, for entry into the cell. Unlike VSV-G, which requires the upregulation of the LDLR by pre-stimulation with a high concentration of cytokines, BaEV receptors are already expressed at basal levels in human HSCs. This allows for gene transfer without the need for activation of HSCs, retaining their stem-ness and multipotency. Verhoeyen et al. showed that BaEv-LVs led to a significantly higher level of transduction compared to VSVG-LVs, without the need for pre-stimulation. BaEV-transduced HSCs also showed long-term engraftment and effective multi-lineage potential in immunocompromised mice using lower amounts of cytokines. Therefore, BaEV-LVs are potentially superior to VSVG-LVs for gene therapy using HSCs. ${ }^{38}$ BaEV-LVs were also recently shown to be more than 10-fold more efficient at transducing primary human B cells compared to VSVG-LVs upon B cell receptor-mediated stimulation. ${ }^{39}$

\section{PARAMYXOVIRAL ENVELOPES}

A well-studied envelope used for pseudotyping comes from the measles virus (MV). ${ }^{40} \mathrm{MV}$ is a singlestranded, negative-sense RNA virus from the Paramyxoviridae family. Humans are the sole natural hosts of MV, which causes measles, a respiratory infection. MV relies on cell-cell fusion for transmission of virus. As with other paramyxoviruses, MV has hemagglutinin $(\mathrm{H})$ on its surface. Although there are eight clades of MV, they share a single $\mathrm{H}$ serotype. MV fusion with the cell membrane is mediated by its fusion protein (F). MV-F is a type 1 membrane protein, which mediates $\mathrm{pH}$ independent fusion by cleavage of its ectodomain into two subunits. MV-H interacts with the cleaved $\mathrm{F}$ trimers through its membrane proximal regions and triggers fusion. Therefore, presence of both $\mathrm{H}-\mathrm{F}$ is essential for formation of fusogenic complexes. Several reports have shown that HIV-based LVs can be pseudotyped effectively with MV-H-F. ${ }^{40,41}$ The cytoplasmic tail of $\mathrm{H}-\mathrm{F}$ complexes interacts with the HIV capsid protein, allowing attachment and fusion of pseudotyped vectors. MV-pseudotyped LVs have shown to be par- ticularly useful for transducing cells of hematopoietic origin. ${ }^{41-43}$ Receptor usage by MV-LVs depends on the strains from which the envelopes are derived. The cellular receptors for $\mathrm{H}$ are CD46 and signaling lymphocyte activation molecule (SLAM) ${ }^{44}$ CD46 is expressed on all human nucleated cells, whereas SLAM is expressed constitutively on CD45RO ${ }^{\text {hi }}$ memory $\mathrm{T}$ cells, immature thymocytes, B cells, macrophages, and dendritic cells (DCs). LVs pseudotyped with envelope from wild-type (WT-MV) or Edmonston vaccine (EdM-MV) strain showed differential usage of receptors across several cell lines: CD46 binding was sufficient for EdM-MV, whereas WT-MV required both CD46 and SLAM on the cell surface. Moreover, for WT-MV, binding of $\mathrm{H}$ proteins to both CD46 and SLAM on the same cell was essential for transduction. ${ }^{44,45}$ The EdM-MV strain is better suited for pseudotyping LVs because it can attach to either CD46 or SLAM, unlike WT-MV, which required both receptors to be present. EdMMV-LVs transduce resting $B$ and $T$ cells significantly more efficiently than VSVG-LVs, and stimulated or activated lymphocytes as efficiently. Resting lymphocytes are notoriously difficult to transduce with VSVG-LVs due to low LDLR expression, and MVLVs provide an excellent alternative. ${ }^{11} \mathrm{MV}-\mathrm{LV}$ s also showed better transduction of DCs, which coincides with high CD46 expression. ${ }^{41,44,46}$ Moreover, the presence of Dendritic Cell-Specific Intracellular adhesion molecule-3-Grabbing Non-integrin (DC-SIGN) on DCs enhances transduction. DC-SIGN is not sufficient cell entry, but rather enhances infection of MV. Furthermore, transduction with MV-LVs did not cause maturation of DCs, unlike VSVGLVs, which increases their utility to transduce DCs. ${ }^{47}$ MV-LVs can also be concentrated using high-speed centrifugation or ultracentrifugation, allowing generation of high-titer vectors preparations. Recently, Mustang-Q column chromatography was used to concentrate MV-LVs, demonstrating their clinical utility. ${ }^{48}$ A remarkable example of highly specific tropism comes from another paramyxovirus, Nipah Virus (NiV). ${ }^{49,50} \mathrm{NiV}$ is a zoonotic virus, with fruit bats as its primary hosts. NiV can infect humans, resulting in multiple, potentially fatal flu-like symptoms as well as encephalitis. NiV envelope is made up of $\mathrm{F}$ and $\mathrm{G}$ glycoproteins. NiV-G binds its receptor, EphrinB2, with high affinity and causes conformational changes that lead to NiV-Fmediated fusion and viral entry. EphrinB2 is expressed on several multipotent and pluripotent cells such as murine embryonic stem cells, human HSCs, and human neural stem cells. Therefore, pseudotyping with NiV-F-G can be of great use, especially to transduce these difficult to transduce 
cells. NiV-F-G can pseudotype LVs efficiently, especially upon deletion of parts of the $\mathrm{F}$ and $\mathrm{G}$ proteins. NiV-LVs transduce multipotent and pluripotent stem cells with high efficiency both in vitro and in vivo. Another unique feature of NiV-LVs is that they are not shunted into the liver sink upon systemic administration in mice, unlike VSVG-LVs. ${ }^{49,50}$

\section{RHABDOVIRAL ENVELOPES}

Rabies virus (RabV) is a single-stranded negative-sense RNA virus from the Rhabdoviridae family. RabV has a broad range of host tropism, including humans, rodents, and bats. RabV causes infections of the central nervous system (CNS) that is often fatal. Like VSV, RabV uses its envelope glycoprotein (RabVG) for attachment to the hostcell surface and for mediating viral entry. The receptors for RabVG are neurotrophic receptor (p75NTR), Neural Cell Adhesion Molecule (NCAM), and nicotinic acetylcholine receptor (nAChR), which are all expressed on neural cells. ${ }^{51}$ Upon binding its receptor, RabV enters the cell via Clathrin-mediated endocytosis that is dependent on the RAB5 protein. During maturation of endosomes to lysosomes, reduction in $\mathrm{pH}$ triggers fusion and entry. Neurotropism of RabVG makes it an attractive envelope to pseudotype LVs for transduction of neuronal cells. RabV-LVs mediate efficient transduction of neurons in vitro and in vivo. ${ }^{52-57}$ RabV-LVs are able to move from the axonal surfaces to the nucleus via retrograde transport mediated by dynein motor complex. RabV-G co-localizes with its receptors, which shuttle to the neuronal soma via retrograde transport, allowing RabV-LVs to access the nucleus. As RabV and VSV are closely related, chimeric proteins created by fusing parts of VSVG and RabVG can also pseudotype LVs. These fusion proteins (FuG-A/B/B2/C/E) show different tropism and retrograde transport efficiencies. In particular, HiRet (FuG-B/B2) and FuG$\mathrm{E}$ are highly efficient at transduction and retrograde transport. ${ }^{57-60}$ The $\mathrm{G}$ protein from a related Rhabdovirus, Mokola virus (MOKV-G), also shows similar ability to pseudotype LVs. MOKV infects cats and other sub-Saharan mammals, but rarely humans. MOKV-G shares antigenic serotype with RabV but shows broader tropism. MOKV-LVs transduce a broad variety of cells in murine brain upon in vivo transduction. ${ }^{19,30,61,62}$ Another viral envelope that has been tested along with RabV is from the highly pathogenic Ebola virus Zaire (EboZ). EboZ belongs to the Filoviridae family, and causes highly morbid hemorrhagic fevers. Primary hosts and reservoirs of EboZ are various species of bats, with primates and humans being end hosts. EboZ is a large negative-sense, single-stranded RNA virus. EboZ envelope consists of heavily glycosylated envelope glycoproteins that mediate both attachment and entry. The putative receptors for EboZ are NPC1 (Niemann-Pick C1, a lysosomal cholesterol transporter) and TIM1 (T-cell Immunoglobulin and Mucin domain 1, a receptor for phosphatidylserine), which are expressed on $\mathrm{T}$ cells and other hematopoietic cells. EboZ-LVs can transduce lung epithelial cells, skin fibroblasts, and epidermal progenitor cells at comparable levels to VSVG-LVs. ${ }^{62,63}$ EboZ-LVs also showed predominantly apical specificity for transduction. However, high levels of cell decay and death hampers their utility. Another concern in using Filoviral envelopes is their high biosafety risk, as they are highly pathogenic.

\section{LYMPHOCYTIC CHORIOMENINGITIS VIRUS ENVELOPE}

Lymphocytic ChorioMeningitis Virus (LCMV) is a negative-sense, single-stranded RNA virus belonging to the family Arenaviridae. The primary hosts and reservoirs of LCMV are mice, but hamsters and other rodents can propagate and transmit the virus. LCMV can also infect dogs, pigs, primates, and even humans. In rodents, LCMV causes inflammation of the meninges due to lymphocytic infiltrates, which can be fatal in mice. LCMV has been well-studied for its ability to induce robust $\mathrm{CD} 8+\mathrm{T}$ cell responses in infected hosts. ${ }^{19,64-66}$ The receptor for LCMV is $\alpha$-dystroglycan, which is expressed mainly on DCs, with low levels of expression on neural cells and hepatocytes. LCMV envelope glycoprotein is cleaved into two subunits: GP1, a peripheral receptor binding subunit, and GP2, an integral membrane protein that anchors the virus and facilitates entry. LCMVLVs can transduce murine DCs, astrocytes, neural progenitors, and glial cells. Although both VSVGLVs and LCMV-LVs can transduce cells of the CNS, their tropisms differ. ${ }^{62,67}$ Upon in vivo injection, LCMV-LVs transduce astrocytes, whereas VSVGLVs transduce neurons. ${ }^{62,67}$ LCMVs can also transduce human and murine hepatocytes. In addition to wide tropism, LCMV-LVs are amenable to concentration of ultracentrifugation and to production using a packaging cell line. ${ }^{64,65}$

\section{BACULOVIRUS ENVELOPE}

Baculoviruses make an intriguing source of envelopes for pseudotyping LVs. Members of the family Baculoviridae infect $>600$ species of invertebrate animals, including arthropods and lepidoptera. Baculoviruses are double-stranded DNA viruses that rely on one envelope glycoprotein for 
attachment and fusion. The most well-studied baculoviral envelope protein is GP64 from Autographa californica. While a single receptor for GP64 has not been identified, it is known that it interacts with phospholipids on mammalian cells and that heparan sulfate is essential for mammalian cell transduction. ${ }^{68-70}$ Homotrimeric GP64 interacts with CD55 in lipid rafts and induces fusion at low $\mathrm{pH}$. The actual mechanism for endocytosis is not clear, and may involve both Clathrin-dependent and Clathrin-independent pathways. LVs pseudotyped with GP64 (GP64-LVs) exhibit broad tropism in mammalian cells. ${ }^{68-70}$ GP64-LVs can transduce BHK, MDCK, HeLa, Huh7, and 293T cells at levels comparable to VSVG-LVs. They show lower transduction of certain cell lines, for example HepG2 and HT1080, than VSVG-LVs do. GP64-LVs are particularly inefficient at transducing cells of hematopoietic origin such as K562, MOLT4, Raji, and CEM cells. Efficient transduction of hepatocytes makes GP64-LVs attractive candidates for gene therapy for liver diseases. Indeed, in vivo injections of GP64-LVs expressing factor VIII result in longterm correction of hemophilia. In addition, GP64LVs were able to transduce airway epithelial cells upon intranasal transduction in mice. ${ }^{71}$ GP64 envelopes are also sufficiently stable to withstand ultracentrifugation and sufficiently less toxic, compared to VSVG, to allow production from a packaging cell line.

\section{ALPHAVIRAL ENVELOPES}

Ross River virus (RRV), Semliki Forest virus (SFV), Chikungunya virus (CHIKV), Sindbis virus (SV), Venezuelan Equine Encephalitis virus (VEEV), and western equine encephalitis virus (WEEV) are all alphaviruses that belong to the family Togaviridae. Alphaviruses are mosquito-borne viruses that infect humans and cause immunoinflammatory manifestations. Alphaviruses are small positive-sense, single-stranded RNA viruses with an icosahedral capsid. The alphavirus envelope is heterodimeric, consisting of two glycoproteins: fusion protein $\mathrm{E} 1$ and receptor binding protein E2. Alphaviral envelopes attach to their target cells and undergo $\mathrm{pH}$-dependent fusion. The cellular receptors of the different Alphaviruses are not known, but heparan sulfate and integrins are thought to play a role in binding. Moreover, C-type lectins DC-SIGN and DC-SIGNR enhance infection of alphaviruses in a glycosylation-dependent manner, without being direct receptors of the envelope. The tropism of $\mathrm{E} 1$ can be altered by point mutations, especially in case of CHIKV. Alphavirus
E1/E2 pseudotyped LVs were shown to exhibit broad tropism in human cell lines. Feline immunodeficiency virus (FIV)-based vectors pseudotyped with RRV and SFV transduced a multitude of adherent and non-adherent cell lines. ${ }^{72}$ These vectors transduced HT1080 and MDA231 cells at efficiencies comparable to VSVG and THP1 and K562 cells at slightly lower efficiencies. RRVand SFV-LVs are not efficient at transduction of other hematopoietic cells, including CD34+ cells. ${ }^{72,73}$ RRV- and SFV-LVs transduce neuroglial cells (astrocytes and oligodendrocytes) better than VSVGLVs upon intracranial injections in mice. ${ }^{74-78}$ Systemic administration of these viruses results in high-level transduction of hepatocytes and Kupffer cells. HIV-based CHIKV-LVs also show broad tropism and transduce multiple cell types at levels comparable to VSVG-LVs. Interestingly, transduction of monocytic THP1 cells enhances significantly upon their differentiation into macrophages. ${ }^{78,79}$ This is possibly due to enhanced expression of viral receptors on their surface. Unlike RRV-LVs, WEEVLVs and VEEV-LVs show much lower titers when pseudotyped. ${ }^{77}$ LVs pseudotyped with SV envelope are a fascinating case that demonstrates the versatility of alphaviruses. As discussed in the sections below, a small peptide inserted in SV envelope glycoprotein allows for attaching a customizable targeting moiety to achieve specific transduction.

\section{ORTHOMYXOVIRAL ENVELOPES}

Influenza viruses such as influenza A-D and fowl plague virus (FPV) belong to the family Orthomyxoviridae. These viruses exhibit diverse tropism, including humans, pigs, horses, and fowl, and cause respiratory infections that can be fatal. Orthomyxoviruses are negative-sense, single-stranded RNA viruses. The envelopes of orthomyxoviruses are made up of three proteins: hemagglutinin (HA), which binds to sialic acid on the host cell, neuraminidase (NA), which releases virions from sialic acid on producer cells and mediates fusion, and M2, a proton channel that regulates $\mathrm{pH}$-dependent viral fusion. HA is proteolytically cleaved into HA1 and HA2 subunits, exposing the fusion domain on HA2. M2 protein is a homotetrameric protein that forms a proton channel, which buffers the cellular compartments. Modulation of $\mathrm{pH}$ by $\mathrm{M} 2$ is essential for both fusion and viral production. During early infection, lowering of $\mathrm{pH}$ by M2 triggers HA-mediated fusion, whereas during viral production, raising of $\mathrm{pH}$ by $\mathrm{M} 2$ protects $\mathrm{HA}$ in nascent viral particles from inactivation. ${ }^{80}$ Because of its tropism, HA can be used to pseudotype LVs for targeting airway 
epithelial cells. LVs pseudotyped with FPV HA show low titers compared to VSVG. ${ }^{81}$ The titers can be enhanced by co-expression of the NA and M2 proteins. While NA expression releases nascent virions efficiently, M2 expression increases the amount of HA incorporated into virions. ${ }^{80} \mathrm{FPV}$ LVs also show preferential transduction of apical surface of airway epithelia, making them useful for lung gene therapy.

\section{TARGETED PSEUDOTYPING}

While the viral envelopes described above have diverse tropism, their specificity depends on the envelope binding properties that have evolved to infect different cell types. In recent years, several groups have developed targeted approaches for transducing specific cell types. These approaches include pseudotyping viruses with engineered envelopes that are more tropic toward a cell type or using proteins or antibodies that bind specifically to the cell of interest. An instance of altering tropism was demonstrated by selection of feline leukemia virus (FeLV) envelope for mutants with altered tropism. A more rational approach was designed to introduce mutations in the FeLV envelope by site-directed mutagenesis, followed by selection of viruses that bind the target cell line. Through this approach, FeLV mutants with altered tropism were identified. ${ }^{82-84}$ A more directed approach for modifying the tropism of envelopes takes advantage of specific ligand-receptor reactions. Such a ligand-based approach was demonstrated by incorporating a protein called heregulin in Mo-MLV virus envelope glycoprotein. Heregulin binds specifically to HER2, a protein expressed on breast cancer cell lines. The chimeric heregulinMoMLV envelopes were able to target breast cancer cells efficiently, demonstrating the feasibility and efficacy of this approach. ${ }^{85} \mathrm{~A}$ similar approach used a CD4-binding Designed Ankyrin Repeat Protein (DARPin) linked to MV-H protein to target LVs to CD4+ T cells. ${ }^{86}$ An even more targeted and perhaps universal approach relies on using antibodies to specific surface proteins to provide targeting. Successful cell targeting has been achieved using a modified Sindbis virus m 168 envelope. The envelope was modified to swap the receptorbinding domain with an Ig-binding ZZ domain derived from staphylococcus protein $\mathrm{A}$, as well as to mutate several residues to inactivate receptorbinding sites. This envelope can be conjugated with an antibody of choice, which allows targetable binding to cells bearing the cognate epitope. The antibody targets the virus to cells expressing its epitope, and the envelope glycoprotein facilitates fusion. This approach was successfully demonstrated using an antibody against CD20 to specifically transduce $\mathrm{B}$ cells in vitro and in vivo. ${ }^{87}$ Similar approaches to target difficult to transduce hematopoietic progenitors were developed using the antibodies for CD133 and ABCG2. ${ }^{88}$ Antibodymediated transduction of total cord blood using antibodies against CD133 or ABCG2 was moderately enhanced in the presence of the antibody, without the need for purification of progenitors. In a similar approach, m168 envelopes conjugated with single-chain antibodies against CD20, DEC205, and P-glycoprotein were used to target B cells, DCs, and melanoma cells, respectively. ${ }^{89}$ A similar approach was used to modify MV-H protein by linking it to an antibody against CD8, to target the vectors to CD8+ T cells. ${ }^{90}$ Retargeted MV-LVs to recognize CD19 or CD20 were shown to be effective in targeting $\mathrm{B}$ cells without activating them. ${ }^{91} \mathrm{MV}$ LVs retargeted to recognize CD133 transduced hematopoietic progenitors efficiently, whereas those retargeted to CD105 transduced endothelial cells efficiently. The flexibility of MV-H protein to accommodate heterologous targeting moieties makes it an attractive option to act as a platform for specifically targeting LVs. ${ }^{92}$ There is a great benefit to uncoupling the antibody and fusogenic parameters in the studies listed above because it allows the two parameters to be optimized separately. However, incorporation of the targeting antibody in a non-covalent manner is not as efficient. Modification of MV-H proteins overcomes these limitations and confers flexibility to target a wide range of desired cell types. As this powerful tool evolves over time, its limitations as well as full potential will be clear. The related paramyxovirus $\mathrm{NiV}$ was also demonstrated to be amenable to incorporation of targeting domains. Recently, retargeting of NiV-G glycoprotein by ablating its binding to EphrinB2, and by incorporating DARPins or scFvs was demonstrated. NiV-LVs retargeted to EpCAM, CD8, CD117, or GluA4 were used successfully. ${ }^{93}$ These reports demonstrate that in the recent years, largely due to the tremendous flexibility of paramyxoviral envelopes, targeting of LVs to specific cell types has become more feasible and efficient.

\section{VECTOR MANUFACTURING CONSIDERATIONS}

The suitability of LVs for clinical gene therapy has prompted advances in their manufacture using good manufacturing practices (GMP). GMP-grade 
vector production requires that vectors preparations be consistently of high quality, in addition to being safe and sterile for clinical use. While packaging cell lines have been tested, transient transfection of $293 \mathrm{~T}$ cells seems to be the method of choice to make clinical grade LVs. Not surprisingly, the majority of the development of GMP-grade vector production has focused on VSVG-LVs. As mentioned before, VSVG-LVs are able to withstand ultracentrifugation and column chromatography for concentration and are resistant to freezethawing, ${ }^{94}$ which is needed for efficient gene transfer in primary cells under GMP conditions. Following transient transfection, a semi-closed manufacturing process is often employed to maintain sterility and avoid vector loss. ${ }^{95,96}$ The vector preparation is treated with Benzonase to remove DNA. This is followed by ultrafiltration, tangential flow filtration, or chromatography using Mustang$\mathrm{Q}$ or similar columns. These methods not only concentrate the virus, but also remove potentially toxic or immunogenic cellular debris from the producer cells. The vector prep then goes through quality control, ensuring that the preparation is sterile, is at sufficient titers, and is replication incompetent. In addition to VSVG-LVs, several other varieties of pseudotyped LVs are amenable to GMP-grade manufacturing. MV-LVs have also been tested for clinical-scale production. ${ }^{48,95}$ As mentioned previously, LCMV-LVs, GP64-LVs, and RabV-LVs are also able to withstand the processes used in GMP production. The notable exceptions are LVs pseudotyped with gammaretroviral envelopes, which cannot withstand ultracentrifugation.

Another important aspect of LV production is the biosafety of the preparation. While LVs carry a risk of insertional oncogenesis, they have undergone several improvements to decrease that risk. These improvements largely concern the vector backbone and are beyond the scope of this review. Moreover, as the majority of the clinically used LVs are derived from HIV, there is also a risk of potential recombination, resulting in replication-competent virus. Progressively, newer generations have minimized this risk by separating different components for vector production onto multiple plasmids. A threeor four-plasmid system is considerably safer than a one- or two-plasmid system. Another important biosafety aspect to consider, particularly regarding heterologous envelopes, is the pathogenicity of the virus from which they are derived. Particularly, the use of envelopes from highly pathogenic viruses such as EboZ, influenza, and alphaviruses needs to be well regulated to minimize safety risk.

\section{CONCLUSION}

Since the advent of LVs, numerous studies have altered their tropism. As reviewed in the preceding sections, these modifications have mostly focused on using envelopes from other viruses. There are two key points that arise from these studies. First, LVs are incredibly versatile, allowing pseudotyping by a diverse set of envelopes derived from viruses across numerous families. Second, the diversity of viral envelopes, including the feasibility of engineering their specificity, allows great customization of LVs for every purpose. These many guises of LVs make them powerful tools for cellspecific transduction.

\section{ACKNOWLEDGMENTS}

We thank Dr. Donald B. Kohn and Dr. Roger P. Hollis for comments and suggestions on the manuscript. A.V.J. and S.S. are supported by Caltech Innovation Initiative (CI2) award and the California Institute of Regenerative Medicine award DISC209123.

\section{AUTHOR DISCLOSURE}

S.S. is a full time employee of PACT Pharma, Inc. A.V.J. has no financial disclosures.

\section{REFERENCES}

1. Blomer U, Naldini L, Kafri T, et al. Highly efficient and sustained gene transfer in adult neurons with a lentivirus vector. J Virol 1997;71:6641-6649.

2. Naldini L, Blomer U, Gallay P, et al. In vivo gene delivery and stable transduction of nondividing cells by a lentiviral vector. Science 1996;272:263-267.

3. Naldini L, Blomer U, Gage FH, et al. Efficient transfer, integration, and sustained long-term expression of the transgene in adult rat brains injected with a lentiviral vector. Proc Natl Acad Sci U S A 1996, 93:11382-11388.

4. Klatzmann D, Champagne E, Chamaret S, et al. Tlymphocyte T4 molecule behaves as the receptor for human retrovirus LAV. Nature 1984;312:767-768.

5. Dalgleish AG, Beverley PC, Clapham PR, et al. The CD4 (T4) antigen is an essential component of the receptor for the AIDS retrovirus. Nature 1984;312: 763-767.

6. Moore JP, Trkola A, Dragic T. Co-receptors for HIV-1 entry. Curr Opin Immunol 1997:9:551-562.

7. Burns JC, Friedmann T, Driever W, et al. Vesicular stomatitis virus $\mathrm{G}$ glycoprotein pseudotyped retroviral vectors: concentration to very high titer and efficient gene transfer into mammalian and 
nonmammalian cells. Proc Natl Acad Sci U S A 1993:90:8033-8037.

8. Finkelshtein D, Werman A, Novick D, et al. LDL receptor and its family members serve as the cellular receptors for vesicular stomatitis virus. Proc Natl Acad Sci U S A 2013;110:7306-7311.

9. Hioki $H$, Kameda $H$, Nakamura $H$, et al. Efficient gene transduction of neurons by lentivirus with enhanced neuron-specific promoters. Gene Ther 2007;14:872-882.

10. Akkina RK, Walton RM, Chen ML, et al. Highefficiency gene transfer into CD34+ cells with a human immunodeficiency virus type 1-based retroviral vector pseudotyped with vesicular stomatitis virus envelope glycoprotein G. J Virol 1996;70: 2581-2585.

11. Amirache F, Levy C, Costa C, et al. Mystery solved: VSV-G-LVs do not allow efficient gene transfer into unstimulated T cells, B cells, and HSCs because they lack the LDL receptor. Blood 2014;123: 1422-1424.

12. Pacchia AL, Adelson ME, Kaul M, et al. An inducible packaging cell system for safe, efficient lentiviral vector production in the absence of HIV1 accessory proteins. Virology 2001;282:77-86.

13. Ni Y, Sun S, Oparaocha I, et al. Generation of a packaging cell line for prolonged large-scale production of high-titer HIV-1-based lentiviral vector. J Gene Med 2005;7:818-834.

14. Farson D, Witt R, McGuinness R, et al. A newgeneration stable inducible packaging cell line for lentiviral vectors. Hum Gene Ther 2001;12: 981-997.

15. DePolo NJ, Reed JD, Sheridan PL, et al. VSV-G pseudotyped lentiviral vector particles produced in human cells are inactivated by human serum. Mol Ther 2000;2:218-222.

16. Sakuma T, Barry MA, Ikeda Y. Lentiviral vectors: basic to translational. Biochem J 2012;443:603618.

17. Hu S, Mohan Kumar D, Sax C, et al. Pseudotyping of lentiviral vector with novel vesiculovirus envelope glycoproteins derived from Chandipura and Piry viruses. Virology 2016;488:162-168.

18. lyer AV, Pahar B, Boudreaux MJ, et al. Recombinant vesicular stomatitis virus-based West Nile vaccine elicits strong humoral and cellular immune responses and protects mice against lethal challenge with the virulent West Nile virus strain LSU-AR01. Vaccine 2009;27:893-903.

19. Wong LF, Azzouz M, Walmsley LE, et al. Transduction patterns of pseudotyped lentiviral vectors in the nervous system. Mol Ther 2004;9: 101-111.

20. Humbert O, Gisch DW, Wohlfahrt ME, et al. Development of third-generation cocal envelope producer cell lines for robust lentiviral gene transfer into hematopoietic stem cells and T-cells. Mol Ther 2016;24:1237-1246.

21. Hwang BY, Schaffer DV. Engineering a serumresistant and thermostable vesicular stomatitis virus $\mathrm{G}$ glycoprotein for pseudotyping retroviral and lentiviral vectors. Gene Ther 2013;20:807815.

22. Kafri T, Blomer U, Peterson DA, et al. Sustained expression of genes delivered directly into liver and muscle by lentiviral vectors. Nat Genet 1997; 17:314-317.

23. Peng KW, Pham L, Ye H, et al. Organ distribution of gene expression after intravenous infusion of targeted and untargeted lentiviral vectors. Gene Ther 2001;8:1456-1463.

24. Sakuma T, De Ravin SS, Tonne JM, et al. Characterization of retroviral and lentiviral vectors pseudotyped with xenotropic murine leukemia virus-related virus envelope glycoprotein. Hum Gene Ther 2010;21:1665-1673.

25. Diaz RM, Bateman A, Emiliusen L, et al. A lentiviral vector expressing a fusogenic glycoprotein for cancer gene therapy. Gene Ther 2000;7: 1656-1663.

26. Jang JE, Shaw K, Yu XJ, et al. Specific and stable gene transfer to human embryonic stem cells using pseudotyped lentiviral vectors. Stem Cells Dev 2006;15:109-117.

27. Bell AJ Jr, Fegen D, Ward M, et al. RD114 envelope proteins provide an effective and versatile approach to pseudotype lentiviral vectors. Exp Biol Med 2010;235:1269-1276.

28. Di Nunzio F, Piovani B, Cosset FL, et al. Transduction of human hematopoietic stem cells by lentiviral vectors pseudotyped with the RD114-TR chimeric envelope glycoprotein. Hum Gene Ther 2007:18:811-820.

29. Germain E, Roullin VG, Qiao J, et al. RD114pseudotyped retroviral vectors kill cancer cells by syncytium formation and enhance the cytotoxic effect of the TK/GCV gene therapy strategy. J Gene Med 2005;7:389-397.

30. Steffens S, Tebbets J, Kramm CM, Lindemann D, Flake A, Sena-Esteves M. Transduction of human glial and neuronal tumor cells with different lentivirus vector pseudotypes. J Neurooncol 2004;70: 281-288.

31. Hanawa $H$, Kelly PF, Nathwani AC, et al. Comparison of various envelope proteins for their ability to pseudotype lentiviral vectors and transduce primitive hematopoietic cells from human blood. Mol Ther 2002;5:242-251.

32. Hanawa H, Hematti P, Keyvanfar K, et al. Efficient gene transfer into rhesus repopulating hematopoietic stem cells using a simian immunodeficiency virus-based lentiviral vector system. Blood 2004;103:4062-4069.

33. Hale CR, Majumdar S, Elmore J, et al. Essential features and rational design of CRISPR RNAs that function with the Cas RAMP module complex to cleave RNAs. Mol Cell 2012;45: 292-302.

34. Schambach A, Galla M, Modlich U, et al. Lentiviral vectors pseudotyped with murine ecotropic envelope: increased biosafety and convenience in preclinical research. Exp Hematol 2006;34:588592.

35. Janssens W, Chuah MK, Naldini L, et al. Efficiency of onco-retroviral and lentiviral gene transfer into primary mouse and human Blymphocytes is pseudotype dependent. Hum Gene Ther 2003;14:263-276.

36. Sandrin V, Muriaux D, Darlix JL, et al. Intracellular trafficking of Gag and Env proteins and their interactions modulate pseudotyping of retroviruses. J Virol 2004;78:7153-7164.

37. Sandrin V, Boson B, Salmon P, et al. Lentiviral vectors pseudotyped with a modified RD114 envelope glycoprotein show increased stability in sera and augmented transduction of primary lymphocytes and CD34+ cells derived from human and nonhuman primates. Blood 2002;100: 823-832.

38. Girard-Gagnepain A, Amirache F, Costa C, et al. Baboon envelope pseudotyped LVs outperform VSV-G-LVs for gene transfer into early-cytokinestimulated and resting HSCs. Blood 2014;124: 1221-1231.

39. Levy C, Fusil F, Amirache F, et al. Baboon envelope pseudotyped lentiviral vectors efficiently transduce human B cells and allow active factor IX B cell secretion in vivo in NOD/SCIDgammac-/- mice. J Thromb Haemost 2016;14:2478-2492.

40. Buchholz CJ, Muhlebach MD, Cichutek K. Lentiviral vectors with measles virus glycoproteinsdream team for gene transfer? Trends Biotechnol 2009:27:259-265.

41. Uhlig KM, Schulke S, Scheuplein VA, et al. Lentiviral protein transfer vectors are an efficient vaccine platform and induce a strong antigenspecific cytotoxic T cell response. J Virol 2015;89: 9044-9060

42. Frecha $C$, Costa $C$, Negre D, et al. Stable transduction of quiescent $T$ cells without induction of cycle progression by a novel lentiviral vector pseudotyped with measles virus glycoproteins. Blood 2008;112:4843-4852.

43. Zhou 0 , Schneider IC, Gallet $M$, et al. Resting lymphocyte transduction with measles virus glycoprotein pseudotyped lentiviral vectors relies on CD46 and SLAM. Virology 2011;413:149-152.

44. Frecha C, Levy C, Costa C, et al. Measles virus glycoprotein-pseudotyped lentiviral vector-mediated gene transfer into quiescent lymphocytes requires binding to both SLAM and CD46 entry receptors. J Virol 2011;85:5975-5985.

45. Funke S, Schneider IC, Glaser S, et al. Pseudotyping lentiviral vectors with the wild-type measles virus glycoproteins improves titer and selectivity. Gene Ther 2009;16:700-705.

46. Kneissl S, Abel T, Rasbach A, et al. Measles virus glycoprotein-based lentiviral targeting vectors that avoid neutralizing antibodies. PloS One 2012;7: e46667.

47. Humbert JM, Frecha C, Amirache Bouafia F, et al. Measles virus glycoprotein-pseudotyped lentiviral 
vectors are highly superior to vesicular stomatitis virus $\mathrm{G}$ pseudotypes for genetic modification of monocyte-derived dendritic cells. J Virol 2012;86: 5192-5203.

48. Marino MP, Panigaj M, Ou W, et al. A scalable method to concentrate lentiviral vectors pseudotyped with measles virus glycoproteins. Gene Ther 2015;22:280-285

49. Witting SR, Vallanda P, Gamble AL. Characterization of a third generation lentiviral vector pseudotyped with Nipah virus envelope proteins for endothelial cell transduction. Gene Ther 2013;20: 997-1005.

50. Palomares K, Vigant F, Van Handel B, et al. Nipah virus envelope-pseudotyped lentiviruses efficiently target ephrinB2-positive stem cell populations in vitro and bypass the liver sink when administered in vivo. J Virol 2013;87:2094-2108.

51. Kobayashi K, Kato S, Inoue K, et al. Altering entry site preference of lentiviral vectors into neuronal cells by pseudotyping with envelope glycoproteins. Methods Mol Biol 2016;1382: 175-186.

52. Mazarakis ND, Azzouz M, Rohll JB, et al. Rabies virus glycoprotein pseudotyping of lentiviral vectors enables retrograde axonal transport and access to the nervous system after peripheral delivery. Hum Mol Genet 2001;10:2109-2121.

53. Hirano M, Kato S, Kobayashi K, et al. Highly efficient retrograde gene transfer into motor neurons by a lentiviral vector pseudotyped with fusion glycoprotein. PloS One 2013;8:e75896.

54. Hislop JN, Islam TA, Eleftheriadou I, et al. Rabies virus envelope glycoprotein targets lentiviral vectors to the axonal retrograde pathway in motor neurons. J Biol Chem 2014;289:1614816163.

55. Kato S, Inoue K, Kobayashi K, et al. Efficient gene transfer via retrograde transport in rodent and primate brains using a human immunodeficiency virus type 1-based vector pseudotyped with rabies virus glycoprotein. Hum Gene Ther 2007;18:11411151.

56. Schoderboeck L, Riad S, Bokor AM, et al. Chimeric rabies SADB19-VSVg-pseudotyped lentiviral vectors mediate long-range retrograde transduction from the mouse spinal cord. Gene Ther 2015;22 357-364.

57. Carpentier DC, Vevis K, Trabalza A, et al. Enhanced pseudotyping efficiency of HIV-1 lentivira vectors by a rabies/vesicular stomatitis virus chimeric envelope glycoprotein. Gene Ther 2012;19: $761-774$

58. Cronin J, Zhang XY, Reiser J. Altering the tropism of lentiviral vectors through pseudotyping. Curr Gene Ther 2005;5:387-398.

59. Kato S, Kobayashi K, Kobayashi K. Improved transduction efficiency of a lentiviral vector for neuron-specific retrograde gene transfer by optimizing the junction of fusion envelope glycoprotein. J Neurosci Methods 2014:227:151-158.
60. Trabalza A, Eleftheriadou I, Sgourou A, et al. Enhanced central nervous system transduction with lentiviral vectors pseudotyped with RVG/HIV1 gp41 chimeric envelope glycoproteins. J Virol 2014;88:2877-2890

61. Colin A, Faideau M, Dufour N, et al. Engineered lentiviral vector targeting astrocytes in vivo. Glia 2009:57:667-679

62. Hachiya A, Sriwiriyanont P, Patel A, et al. Gene transfer in human skin with different pseudotyped HIV-based vectors. Gene Ther 2007;14 648-656.

63. Medina MF, Kobinger GP, Rux J, et al. Lentiviral vectors pseudotyped with minimal filovirus envelopes increased gene transfer in murine lung. Mol Ther 2003;8:777-789.

64. Dylla DE, Xie L, Michele DE, et al. Altering alphadystroglycan receptor affinity of LCMV pseudotyped lentivirus yields unique cell and tissue tropism. Genet Vaccines Ther 2011;9:8.

65. Zhang C, Hu B, Xiao L, et al. Pseudotyping lentiviral vectors with lymphocytic choriomeningitis virus glycoproteins for transduction of dendritic cells and in vivo immunization. Hum Gene Ther Methods 2014:25:328-338.

66. Limberis MP, Bell CL, Heath J, et al. Activation of transgene-specific $T$ cells following lentivirusmediated gene delivery to mouse lung. Mol Ther 2010;18:143-150.

67. Watson DJ, Kobinger GP, Passini MA, et al. Targeted transduction patterns in the mouse brain by lentivirus vectors pseudotyped with VSV, Ebola, Mokola, LCMV, or MuLV envelope proteins. Mol Ther 2002:5:528-537.

68. Kumar M, Bradow BP, Zimmerberg J. Largescale production of pseudotyped lentiviral vectors using baculovirus GP64. Hum Gene Ther 2003;14:67-77.

69. Schauber CA, Tuerk MJ, Pacheco CD, et al. Lentiviral vectors pseudotyped with baculovirus gp64 efficiently transduce mouse cells in vivo and show tropism restriction against hematopoietic cell types in vitro. Gene Ther 2004;11: $266-275$

70. Sinn PL, Burnight ER, Hickey MA, et al. Persistent gene expression in mouse nasal epithelia following feline immunodeficiency virus-based vector gene transfer. J Virol 2005;79:12818-12827.

71. Buckley SM, Howe SJ, Sheard V, et al. Lentiviral transduction of the murine lung provides efficient pseudotype and developmental stage-dependent cell-specific transgene expression. Gene Ther 2008:15:1167-1175

72. Kang $Y$, Stein CS, Heth JA, et al. In vivo gene transfer using a nonprimate lentiviral vector pseudotyped with Ross River virus glycoproteins. J Virol 2002:76:9378-9388.

73. Kahl CA, Marsh J, Fyffe J, et al. Human immunodeficiency virus type 1-derived lentivirus vectors pseudotyped with envelope glycoproteins derived from Ross River virus and Semliki Forest virus. $J$ Virol 2004;78:1421-1430.

74. Jakobsson J, Nielsen TT, Staflin K, et al. Efficient transduction of neurons using Ross River glycoprotein-pseudotyped lentiviral vectors. Gene Ther 2006;13:966-973.

75. Kahl CA, Pollok K, Haneline LS, et al. Lentiviral vectors pseudotyped with glycoproteins from Ross River and vesicular stomatitis viruses: variable transduction related to cell type and culture conditions. Mol Ther 2005;11:470-482.

76. Kolokoltsov AA, Weaver SC, Davey RA. Efficient functional pseudotyping of oncoretroviral and lentiviral vectors by Venezuelan equine encephalitis virus envelope proteins. J Virol 2005;79:756763

77. Poluri A, Ainsworth R, Weaver SC, et al. Functional pseudotyping of human immunodeficiency virus type 1 vectors by Western equine encephalitis virus envelope glycoprotein. J Virol 2008;82: 12580-12584.

78. Salvador B, Zhou Y, Michault A, et al. Characterization of Chikungunya pseudotyped viruses: Identification of refractory cell lines and demonstration of cellular tropism differences mediated by mutations in E1 glycoprotein. Virology2009; 393:33-41.

79. Eleftheriadou I, Dieringer M, Poh XY, et al. Selective transduction of astrocytic and neuronal CNS subpopulations by lentiviral vectors pseudotyped with Chikungunya virus envelope. Biomaterials 2017:123:1-14.

80. McKay T, Patel M, Pickles RJ, et al. Influenza M2 envelope protein augments avian influenza hemagglutinin pseudotyping of lentiviral vectors. Gene Ther 2006;13:715-724.

81. Patel M, Giddings AM, Sechelski J, et al. High efficiency gene transfer to airways of mice using influenza hemagglutinin pseudotyped lentiviral vectors. J Gene Med 2013;15:51-62.

82. Schlecht-Louf G, Mangeney M, El-Garch H, et al. A targeted mutation within the feline leukemia virus (FeLV) envelope protein immunosuppressive domain to improve a canarypox virus-vectored FeLV vaccine. J Virol 2014;88:992-1001.

83. Bupp K, Roth MJ. Targeting a retroviral vector in the absence of a known cell-targeting ligand. Hum Gene Ther 2003:14:1557-1564.

84. Sarangi A, Bupp K, Roth MJ. Identification of a retroviral receptor used by an envelope protein derived by peptide library screening. Proc Natl Acad Sci U S A 2007;104:11032-11037.

85. Snitkovsky S, Young JA. Targeting retroviral vector infection to cells that express heregulin receptors using a TVA-heregulin bridge protein. Virology 2002;292:150-155

86. Zhou 0, Uhlig KM, Muth A, et al. Exclusive transduction of human $\mathrm{CD} 4+\mathrm{T}$ cells upon systemic delivery of CD4-targeted lentiviral vectors. $J$ Immunol 2015;195:2493-2501. 
87. Yang L, Bailey L, Baltimore D, et al. Targeting lentiviral vectors to specific cell types in vivo. Proc Natl Acad Sci U S A 2006;103:1147911484.

88. Zhang X, Roth MJ. Antibody-directed lentiviral gene transduction in early immature hematopoietic progenitor cells. J Gene Med 2010;12: 945-955.

89. Maurice M, Verhoeyen E, Salmon P, et al. Efficient gene transfer into human primary blood lymphocytes by surface-engineered lentiviral vectors that display a $T$ cell-activating polypeptide. Blood 2002;99:2342-2350.

90. Zhou Q, Schneider IC, Edes I, et al. T-cell receptor gene transfer exclusively to human $\mathrm{CD} 8(+)$ cells enhances tumor cell killing. Blood 2012;120:43344342.

91. Kneissl S, Zhou 0, Schwenkert M, et al. CD19 and CD2O targeted vectors induce minimal activation of resting B lymphocytes. PloS One 2013;8:e79047.

92. Anliker B, Abel T, Kneissl S, et al. Specific gene transfer to neurons, endothelial cells and hematopoietic progenitors with lentiviral vectors. Nat Methods 2010;7:929-935.

93. Bender RR, Muth A, Schneider IC, et al. Receptortargeted Nipah virus glycoproteins improve celltype selective gene delivery and reveal a preference for membrane-proximal cell attachment. PLoS Pathog 2016;12:e1005641.
94. Kutner RH, Zhang XY, Reiser J. Production, concentration and titration of pseudotyped HIV1-based lentiviral vectors. Nat Protoc 2009;4: 495-505.

95. Merten OW, Hebben M, Bovolenta C. Production of lentiviral vectors. Mol Ther Methods Clin Dev 2016;3:16017.

96. Ausubel LJ, Hall C, Sharma A, et al. Production of CGMP-grade lentiviral vectors. Bioprocess Int 2012;10:32-43.

Received for publication May 16, 2017; accepted after revision August 27, 2017.

Published online: September 4, 2017. 